\title{
Observations of electron vorticity in the inner plasma sheet
}

\author{
C. Gurgiolo ${ }^{1}$, M. L. Goldstein ${ }^{2}$, A. F. Viñas ${ }^{2}$, W. H. Matthaeus ${ }^{3}$, and A. N. Fazakerley ${ }^{4}$ \\ ${ }^{1}$ Bitterroot Basic Research, Hamilton, MT, USA \\ ${ }^{2}$ Geospace Physics Laboratory, Code 673, NASA Goddard Space Flight Center, Greenbelt, MD, USA \\ ${ }^{3}$ Bartol Research Foundation, University of Delaware, Newark, DE, USA \\ ${ }^{4}$ Mullard Space Science Laboratory, University College London, Holmbury St. Mary Dorking, Surrey RH5 6NT, UK
}

Received: 9 June 2011 - Revised: 11 August 2011 - Accepted: 30 August 2011 - Published: 1 September 2011

\begin{abstract}
From a limited number of observations it appears that vorticity is a common feature in the inner plasma sheet. With the four Cluster spacecraft and the four PEACE instruments positioned in a tetrahedral configuration, for the first time it is possible to directly estimate the electron fluid vorticity in a space plasma. We show examples of electron fluid vorticity from multiple plasma sheet crossings. These include three time periods when Cluster passed through a reconnection ion diffusion region. Enhancements in vorticity are seen in association with each crossing of the ion diffusion region.
\end{abstract}

Keywords. Magnetospheric physics (Plasma sheet) - Space plasma physics (Magnetic reconnection)

\section{Introduction}

There are multiple processes known to occur in the inner plasma sheet that are thought to produce vorticity $(\nabla \times \mathbf{V})$ in the electron fluid velocity. These include both $\mathrm{x}$-line reconnection (Matthaeus, 1982; Matthaeus and Lamkin, 1986) and magnetic bubbles (Birn et al., 2004). To this we might also add bursty bulk flows (BBF), which although not explicitly linked to vorticity, must generate vorticity along the velocity shear boundary (if not throughout the entire flow region).

The launch of Cluster has made possible estimates of the vorticity in the electron fluid velocity. Using the technique reported in Gurgiolo et al. (2010), we have looked for evidence of vorticity in several tail crossings. These crossings include three time periods from two crossings during which Eastwood et al. (2010) report that the Cluster spacecraft found signatures of passing through the ion diffusion region of an $\mathrm{x}$-line reconnection event.

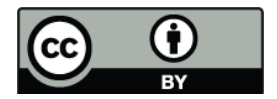

Correspondence to: C. Gurgiolo (chris@gurgiolo.com)
In this paper we will look at occurrences of vorticity in the inner plasma sheet and will endeavor to determine their cause without attempting to trace their origin back to a specific source or mechanism. However, when investigating the three time periods identified by Eastwood et al. (2010) as containing ion jetting from an $\mathrm{x}$-line, we are able to identify an generation mechanism. Our analysis shows localized bursts in vorticity in all of the crossings analyzed. Multiple bursts were not unusual and each of the $\mathrm{x}$-line reconnection events were associated with enhanced vorticity. In all cases vorticity is seen in association with the appearance of fieldaligned electron beams, which can be either mono- or bidirectional and it is not unusual to find the two cases mixed.

\section{Data}

This study uses data from four Cluster experiments. Electron data from the Plasma Electron And Current Experiment (PEACE) forms the primary data set while data from the Fluxgate Magnetometer (FGM); Electric Field and Waves (EFW) and Waves of High frequency and Sounder for Probing of Electron density by Relaxation (WHISPER) experiments are used to characterize the local environment and to provide necessary input to the calculation of moments of the electron distribution function. Below, we provide a brief description of the PEACE experiment as well as how data from the other experiments are used.

PEACE consists of two hemispherical electrostatic analyzers on each of the Cluster satellites (Johnstone et al., 1997). The two analyzers, designated HEEA (High Energy Electrostatic Analyzer) and LEEA (Low Energy Electrostatic Analyzer) are separated by $180^{\circ}$ in phase on the spacecraft and differ only in their geometric factors (HEEA's being the larger of the two). Despite their acronyms, both can cover the energy range from $0.6 \mathrm{eV}$ to $26 \mathrm{keV}$. We include data only from HEEA in this paper. The analyzers' field of view are

Published by Copernicus Publications on behalf of the European Geosciences Union. 
perpendicular to the spacecraft spin axis which is approximately perpendicular to the GSE ecliptic. Each covers $180^{\circ}$ of polar angle in 12 sectors. The full $360^{\circ}$ of azimuth is covered in one rotation of the spacecraft thus allowing a threedimensional snapshot of the electron distribution to be accumulated once per spin $(\sim 4 \mathrm{~s})$, or once every $2 \mathrm{~s}$ if the energy ranges of HEEA and LEEA are identical.

Because of telemetry restrictions, PEACE generally returns only a subset of the total data collected on-board. Exactly what is returned depends on the instrument mode, which can be separately commanded for both analyzers on each of the four spacecraft. The telemetry rate determines the cadence at which full three-dimensional distributions are downloaded. During the time intervals used in this paper, all spacecraft were operating in burst mode and PEACE was returning 3-D distributions every four seconds. The spacecraft were either returning data in 3DXP1 mode (30 energy steps by 32 azimuth sectors by 6 polar zones per spin) on all four spacecraft, or were returning data in CPXP1 mode on $\mathrm{C} 1$ and C3 and CPX1 mode (34 energy steps by 32 azimuth sectors by 12 polar zones per spin) on $\mathrm{C} 2$ and $\mathrm{C} 4$. The basic difference between the two modes is the higher polar resolution in CPX1. The energy range covered in both modes was from approximately $35 \mathrm{eV}$ to $10 \mathrm{keV}$.

Data from PEACE provide a full description of the local electron environment at each spacecraft. The electron plasma is characterized by the first three electron moments (density, velocity, temperature) of the electron velocity distributions (eVDF). The velocity moments are used as input into an algorithm to compute a set of spatial parameters within the volume formed by the four spacecraft. The parameters include estimates of both the electron compression and vorticity. The spin-averaged spacecraft potential data is obtained from EFW and is used to correct the energy bin limits of the PEACE energy steps prior to computing the moments. WHISPER provides an accurate estimate of the electron density through resonance sounding. Active sounding which consists of a set of stepped frequency transmissions, however, distorts the spacecraft potential which in turn distorts the computed moments. The sounding occurs within a fraction if a spin period and the moments computed during those spins are dropped and replaced by linearly interpolated values using moments on either side of the time gap this causes in the data. Magnetic field data is used to characterize the local plasma environment and to rotate moments into a fieldaligned frame of reference. All data other than the PEACE high-resolution data were obtained from the Cluster Active Archive (CAA).

Events for analysis were selected based on four criteria:

- the spacecraft were crossing the plasma sheet and included a neutral sheet crossing,

- the spacecraft were in a reasonable tetrahedral configuration (the tetrahedron quality factor (Robert et al., 1998) was above 2),
- all requisite experiments were operational,

- all spacecraft were using burst mode telemetry.

The burst mode requirement ensures a time contiguous set of velocity moments which is needed to avoid any significant errors in the spatial derivatives due to time aliasing.

Between 2001 and 2006 there were about 100 events which met these criteria of which we looked at 20 . With the exception of two events which included known crossings of the ion diffusion region, these were selected randomly from the data available.

\section{Moments and spatial derivatives}

The numerical method used to estimate the electron moments and algorithm used to compute the spatial derivatives of the fluid velocity have been covered in detail in Gurgiolo et al. (2010). That paper also includes discussions on possible sources of error and limitations associated with the results of the computations - we refer the reader there for details. Below we discuss items unique to this analysis which were not covered in the paper. For completeness we also present a brief overview of method used to compute the spatial derivatives from the plasma moments.

One problem not addressed in Gurgiolo et al. (2010) because it did not present a major concern in the solar wind upstream of the magnetosphere is the effect of the Active Spacecraft Potential Control experiment (ASPOC). ASPOC uses an ion emitter to reduce the spacecraft potential. Issues arose here because ASPOC was running on only C 3 and $\mathrm{C} 4$ during some of the analyzed intervals and, at times, during only a subset of the total interval. Consequently, the spacecraft potentials were much lower on $\mathrm{C} 3$ and $\mathrm{C} 4$ than on $\mathrm{C} 1$ and $\mathrm{C} 2$ and that in turn created significant differences in the electron moments, especially the density, simply because the analyzers were effectively sampling different energy ranges. The same effect occasionally occurs even when ASPOC is off due to the large variations in density encountered in a tail crossing. This situation creates large and, often times, narrow fluctuations in the spacecraft potential that might not appear simultaneously on all four spacecraft.

To ensure that all the moments derived on each spacecraft covered the same energy range for the entire time interval analyzed, we set the lower energy limit used in the integrals to $30 \mathrm{eV}$ after performing the potential correction. (The lowest spacecraft potentials with and without ASPOC are about $-6 \mathrm{eV}$ and the lowest energy step being returned by HEEA is about $36 \mathrm{eV}$.) This ensures that all moments within an interval were estimated within a fixed energy range. The same range is used when ASPOC is off on all spacecraft.

Once the moments have been computed on each spacecraft, spatial derivatives of the fluid velocity can be constructed which allows for estimates of both the plasma compression $(\nabla \cdot \mathbf{V})$ and vorticity $(\nabla \times \mathbf{V})$. Spatial derivatives of a 
Table 1. Time intervals included in study.

\begin{tabular}{ccccccc}
\hline Event & Time interval & CPXP1 & CPX1 & ASPOC ON & QGM & $<$ SEP $>\mathrm{km}$ \\
\hline 1 & 31 Jul 2001, 21:20 to 01:10 & ALL & & & 2.99 & 1900 \\
2 & 18 Aug 2002, 15:15 to 18:15 & C1/C3 & C2/C4 & C3/C4 & 2.99 & 3700 \\
3 & 6 Sep 2002, 13:40 to 16:40 & C1/C3 & C2/C4 & C3/C4 & 2.95 & 4000 \\
4 & 26 Oct 2002, 08:55 to 11:55 & C1/C3 & C2/C4 & & 2.88 & 4500 \\
5 & 29 Jul 2003, 16:05 to 19:05 & C1/C3 & C2/C4 & & 2.87 & 230 \\
6 & 14 Aug 2003, 14:55 to 17:55 & C1/C3 & C2/C4 & & 2.85 & 230 \\
7 & 24 Sep 2003, 12:25 to 15:25 & C1/C3 & C2/C4 & & 2.91 & 250 \\
\hline
\end{tabular}

scalar quantity $Q$ can be estimated provided that it is known at a minimum of 4 non-coplanar spatial locations, a condition met by the four Cluster spacecraft. (A vector is just a set of three scalar components.) With only four data points we are limited to assuming a linear variation of the quantity across the volume defined by the spacecraft of the form:

$Q_{i}=a_{i}+b_{i} X_{j}+c_{i} Y_{j}+d_{i} Z_{j}$

where $Q$ is the quantity being fit, $i$ is the component $(x, y$, or $z), j$ is the spacecraft, $(a, b, c, d)$ are a set of unknown coefficients, and $(X, Y, Z)$ are the spacecraft GSE position coordinates. For any $Q$ there are 4 equations of the form (1), one per spacecraft. By way of an explicit example consider the solutions for $V_{\mathrm{x}}$. (Solutions for $V_{\mathrm{y}}$ and $V_{z}$ are similar.) This is given by the coupled set of equations:

$$
\begin{aligned}
& C 1: V_{\mathrm{x} 1}=a_{\mathrm{x}}+b_{\mathrm{x}} X_{1}+c_{\mathrm{x}} Y_{1}+d_{\mathrm{x}} Z_{1} \\
& C 2: V_{\mathrm{x} 2}=a_{\mathrm{x}}+b_{\mathrm{x}} X_{2}+c_{\mathrm{x}} Y_{2}+d_{\mathrm{x}} Z_{2} \\
& C 3: V_{\mathrm{x} 3}=a_{\mathrm{x}}+b_{\mathrm{x}} X_{3}+c_{\mathrm{x}} Y_{3}+d_{\mathrm{x}} Z_{3} \\
& C 4: V_{\mathrm{x} 4}=a_{\mathrm{x}}+b_{\mathrm{x}} X_{4}+c_{\mathrm{x}} Y_{4}+d_{\mathrm{x}} Z_{4}
\end{aligned}
$$

that can be solved using any number of common analysis techniques.

Using the solutions for the coefficients to the component velocity equations the compression and vorticity are given as:

$$
\nabla \cdot \mathbf{V}=b_{\mathrm{x}}+c_{\mathrm{y}}+d_{\mathrm{z}}
$$

$\nabla \times \mathbf{V}=\left(c_{\mathrm{z}}-d_{\mathrm{y}}\right) \widehat{x}+\left(d_{\mathrm{x}}-b_{\mathrm{z}}\right) \widehat{y}+\left(b_{\mathrm{y}}-c_{\mathrm{x}}\right) \widehat{z}$

Note that with linear solutions there is no position dependence in either the compression or the vorticity.

\section{Observations}

We present data from seven out of the 20 separate crossings of the inner plasma sheet that we have looked at. (Those events not included all exhibit similar features to those discussed below.) Pertinent information on the seven events included in the paper are listed in Table 1 which includes the crossing times, an identifying event number for each crossing, the PEACE data product used to estimate the moments on each spacecraft, the spacecraft on which ASPOC was active for all or part of the event, the spacecraft tetrahedron quality factor and the average spacecraft separation. The tetrahedron quality factors show that the spacecraft were in a good tetrahedral configuration during each event. With the exception of events 2 and 4, the crossings were chosen at random. Events 2 and 4 were specifically selected because they contain time intervals identified in Table 2 of Eastwood et al. (2010) during which it appeared that one or more of the spacecraft were crossing the ion diffusion region of an $\mathrm{x}$ line reconnection event. In all the events, the spacecraft were moving predominately southward through the plasma sheet.

Figures 1 and 2 show the basic plasma and field characteristics across the intervals of events 2 and 4 . The ion diffusion regions identified by Eastwood et al. (2010) are delimited by the pairs of blue lines. The most prominent features in the plots are the bursts in the vorticity (panel 5). There are three distinct bursts in Fig. 1 centered at 17:05, 17:32 and 18:05 UT and one in Fig. 2 centered at 19:35 UT. Of the four, three cover times when the spacecraft traversed ion diffusion regions associated with reconnection. It is interesting that there is no mention in Eastwood et al. (2010) of any crossing of an ion diffusion region associated with the last burst of vorticity in Fig. 1. That, however, should not be taken to imply that the vorticity is not reconnection related but simply that the crossing did not meet all the criteria used by Eastwood et al. (2010) for identifying an ion diffusion region.

As seen in the figures, the bursts in vorticity are associated with regions of enhanced flow. The flows are often, but not always, seen in surrounding large scale rotations in the magnetic field commonly associated with neutral sheet crossings. The lack of a significant rotation associated with a vorticity burst (as in the second burst in Fig. 1) suggests that the flow exists for some distance on either side of the neutral sheet - as we will show later at least on the order of the spacecraft separation. As such it is probably not unusual to see reconnection associated vorticity without actually observing signatures the ion diffusion region.

Similar bursts of vorticity are seen on each of the other inner plasma sheet crossings noted in Table 1. Figure 3 shows 


\section{Cluster C1 Overview Plots}

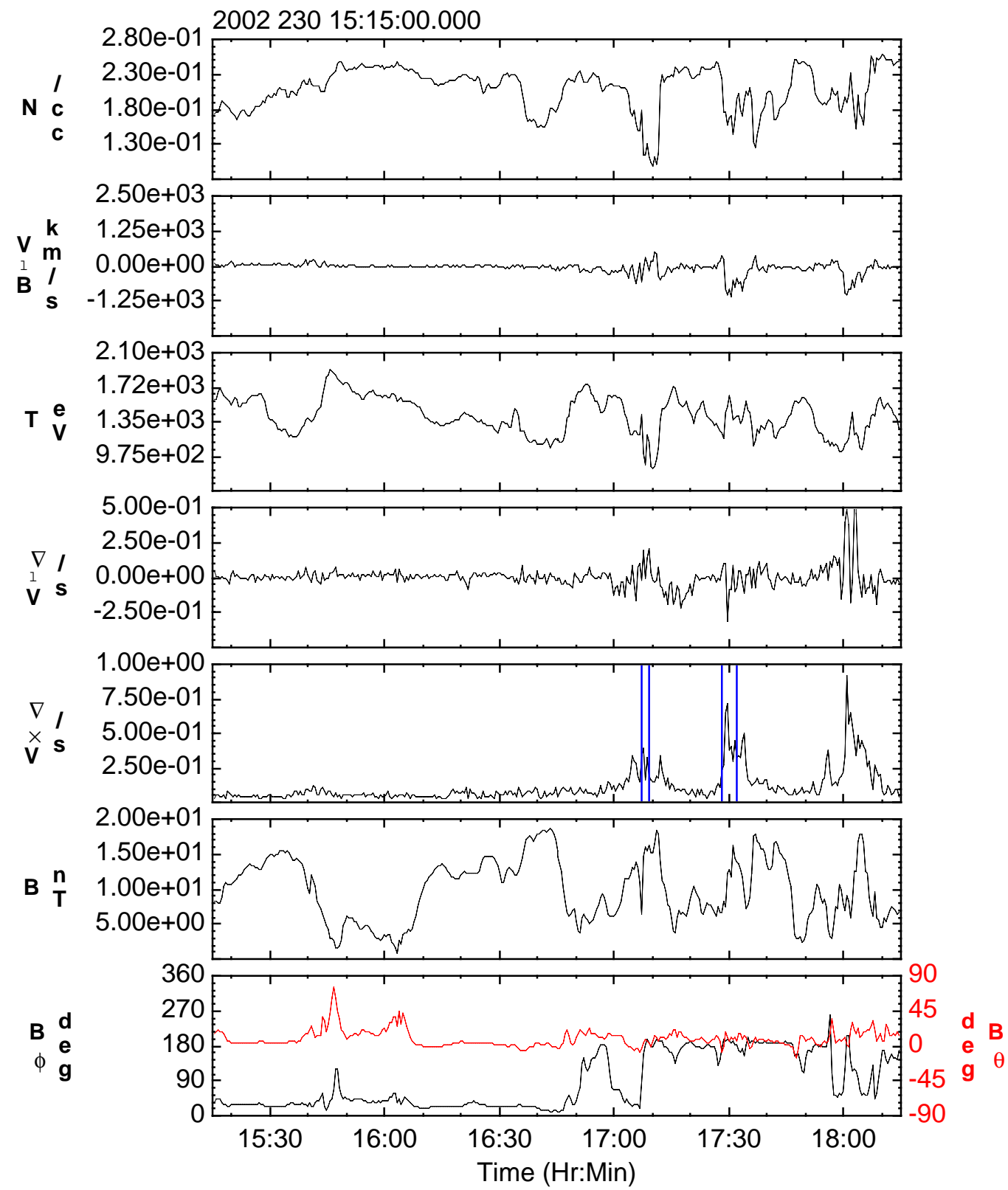

Fig. 1. Overview of the tail crossing on 17 August 2002 (Event 2). From top to bottom are shown the electron density, parallel velocity, temperature, divergence of the velocity, magnitude of the vorticity, total magnetic field, and the polar angles of the magnetic field vector in GSM. With the exception of the divergence and vorticity, which use all four spacecraft, the data shown were obtained from C1. Blue lines in panel 5 demarcate times when the spacecraft made known crossing of an ion diffusion region (Eastwood et al., 2010).

just the magnitude of the vorticity for those events in Table 1 not shown in Figs. 1 and 2. We re-emphasize that all of the listed events, except for 2 and 4, were randomly selected based on the criteria that the spacecraft were in burst mode and were passing through the inner plasma sheet. Events were taken from different years to allow for a variety of spacecraft separations (refer to Table 1). From the events looked at it would seem reasonable to assume that vorticity is a fairly ubiquitous feature of the inner plasma sheet.

In all cases the observed vorticity is associated with times when the spacecraft enters into regions of enhanced velocity. The regions are characterized by mono- and/or bi-directional field-aligned beams with bi-directional beams being the most prevalent. Examples are shown in Figs. 3 and 4. 


\section{Cluster C1 Based Overview Plots}

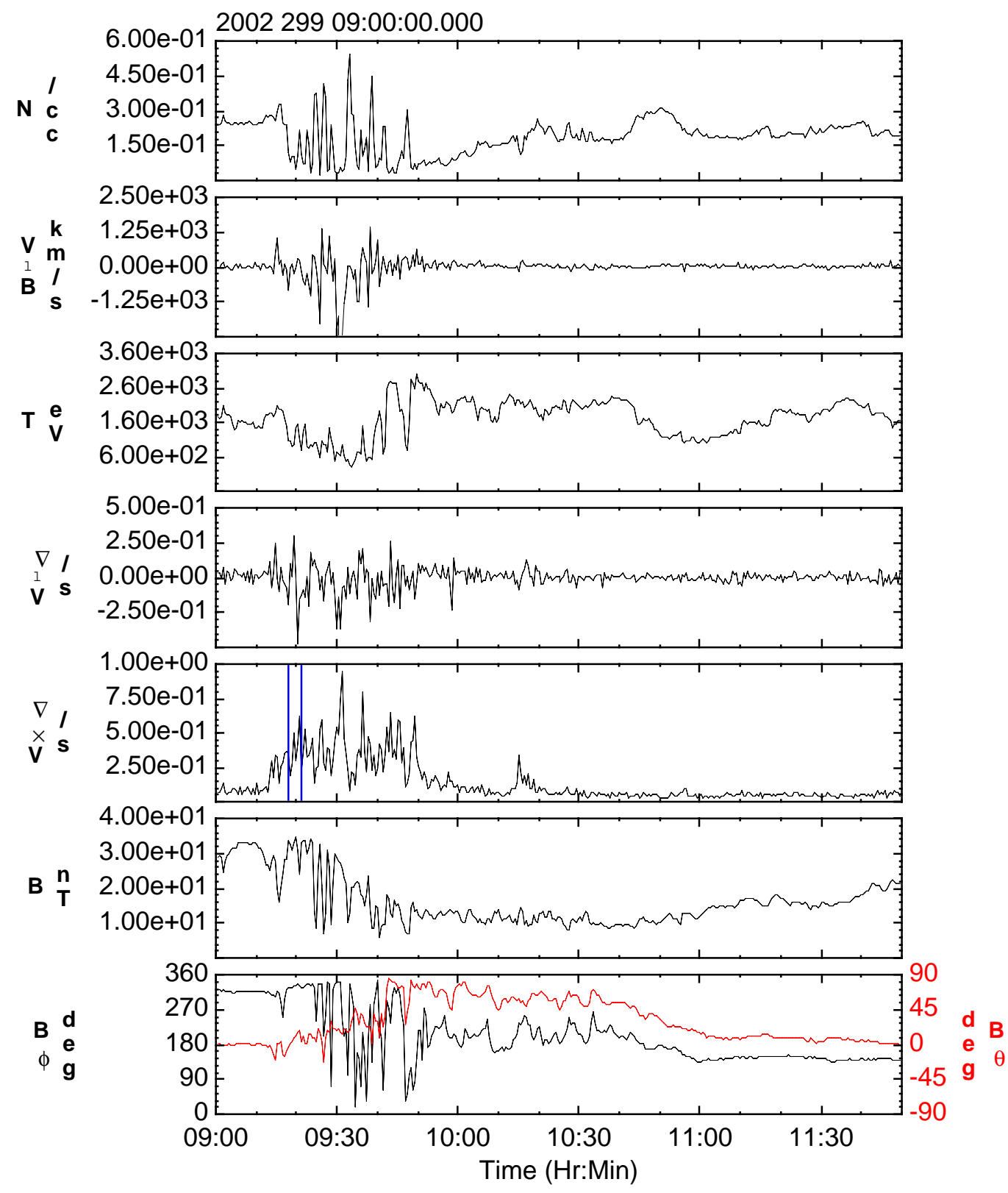

Fig. 2. Overview of tail crossing on 17 October 2002 (Event 4) using the same format as in Fig. 1. Again, with the exception of the divergence and vorticity, which use all four spacecraft, the data shown were obtained from $\mathrm{C} 1$.

Since the format used in the figures is not a common one, we provide a brief description here. We characterize the VDFs through a series of phi-theta (PT) plots. Each plot shows a slice of the eVDF at a constant energy as a function of phi and theta in the PEACE instrument frame of reference. In this frame of reference phi represents the analyzer phase angle and theta the analyzer polar angle. At a phase angle of $0^{\circ}$ the plane defined by the spacecraft spin axis and the normal to the analyzer aperture contains the sun. A theta an- gle of $90^{\circ}$ points along the spacecraft spin axis which makes an angle of about $5^{\circ}$ with $-Z$ GSE. Projections of the head and tail of the magnetic field vector in this coordinate system are included in the plot as a solid circle and triangle, respectively. The projections are made using spin-averaged magnetic field data and as such it is not unusual for the projections to be slightly off from obvious field aligned features. (Ideally the projections should be computed from the magnetic field averaged over the time during which the analyzer 
(a)

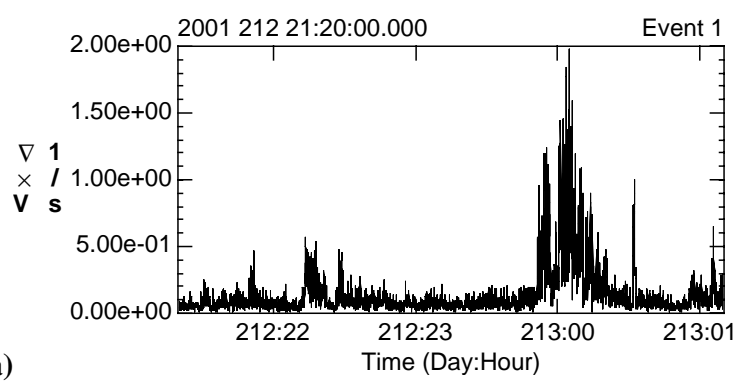

(b)
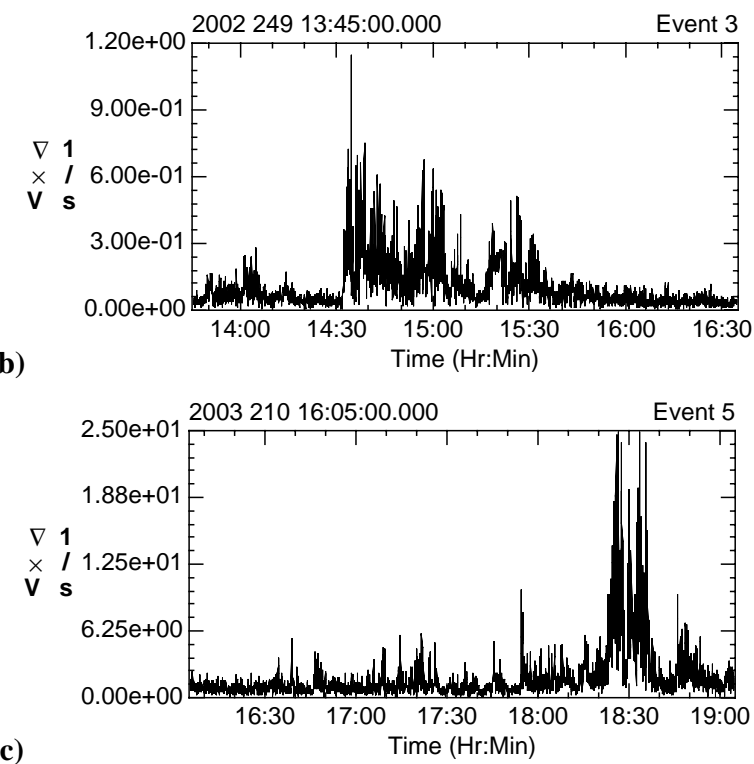

(c)

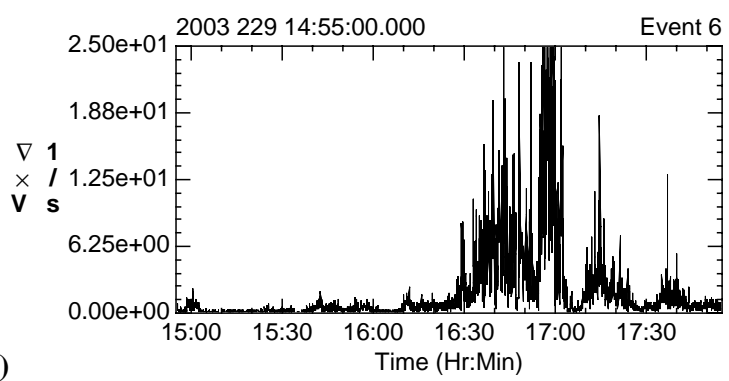

(d)

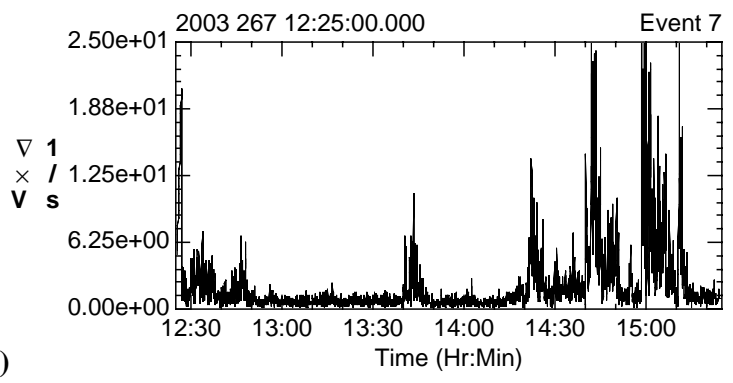

Fig. 3. Plots of the magnitude of the vorticity for five of the inner plasma sheet crossing events listed in Table 1 . The event numbers are given at the upper right of each plot. The plots show that vorticity is a common feature of the inner plasma sheet, often occurring in sets of multiple bursts. spends scanning the field aligned population and not over the entire spin, but we have not done that in these plots.) The plotted data have been smoothed by fitting with a Spherical Harmonic function.

Figure 4 shows details of $5 \mathrm{eVDFs}$ taken on $\mathrm{C} 1$ from within the first burst of vorticity in Fig. 1. All data is shown in units of $\mathrm{s}^{3} \mathrm{~cm}^{-6}$. Each column of PT plots shows data from multiple energy steps for a single eVDF. The PT plots within a column cover the energy range 220 and $7732 \mathrm{eV}$ showing data from every other energy step in the analyzer sweep. Successive columns of plots show data from every other acquired eVDF over the interval 17:07:14 to 17:07:46 UT. The beginning accumulation time for each eVDF is shown above the top plot in the column. The PT plots are individually autoscaled, which allows features that may be suppressed or lost when using a broad scaling range to be readily discernible - a disadvantage is that one is unable to easily intercompare color based intensities between individual plots. Autoscaling sometimes also has the disadvantage of making noise appear as a significant signal. In general, unorganized data such as that lower energy steps in the middle column is noise. The scaling range used for each plot is given directly above it.

The time period covered in the figure begins shortly after the spacecraft enters the ion diffusion and just prior to a crossing of the neutral sheet. The crossing into the neutral sheet begins in the lower portion of the third column of plots at about the energy step where the organized flow is lost. Bidirectional field-aligned beams, which are seen as local enhancements in the eVDF centered on both of the magnetic field projections, appear on the approach to the neutral sheet and continue after its crossing. As seen in the upper plots in column 3, the flow switches to mono-directional beams just before entering neutral sheet. The actual neutral sheet crossing can be very cleanly seen in the projections of the magnetic field with the projections very nearly switching positions between the first and last column.

While most of the enhancements in velocity are the result of bi-directional beams this is not always the case as is shown, for example, in Fig. 5 where the flow results from a complex and dynamic mixture of mono- and bi-directional beams. This is a series of PT plots taken at a fixed energy during the the large vorticity burst beginning just before 17:00 UT in the third panel from the top in Fig. 3. Displaying just a single eVDF energy step allows for a larger interval of time to be displayed. Time in the figure goes from left to right and top to bottom with the start accumulation time of each eVDF given above the plots. The plots cover about $2 \mathrm{~min}$. The figure illustrates the complexity sometimes seen in the electron beams. Note the three successive plots beginning at 16:56:06 UT: The first shows a set of bi-directional beams that switches to a predominately anti-parallel beam in the next plot and then to a parallel beam in the last plot. Such rapidly changing flow patterns lead to large variability in the bulk flow including reversals in the parallel flow direction. 


\section{CLUSTERII.CLUSTER-1.PEACE.3DX1.CPXP1H}

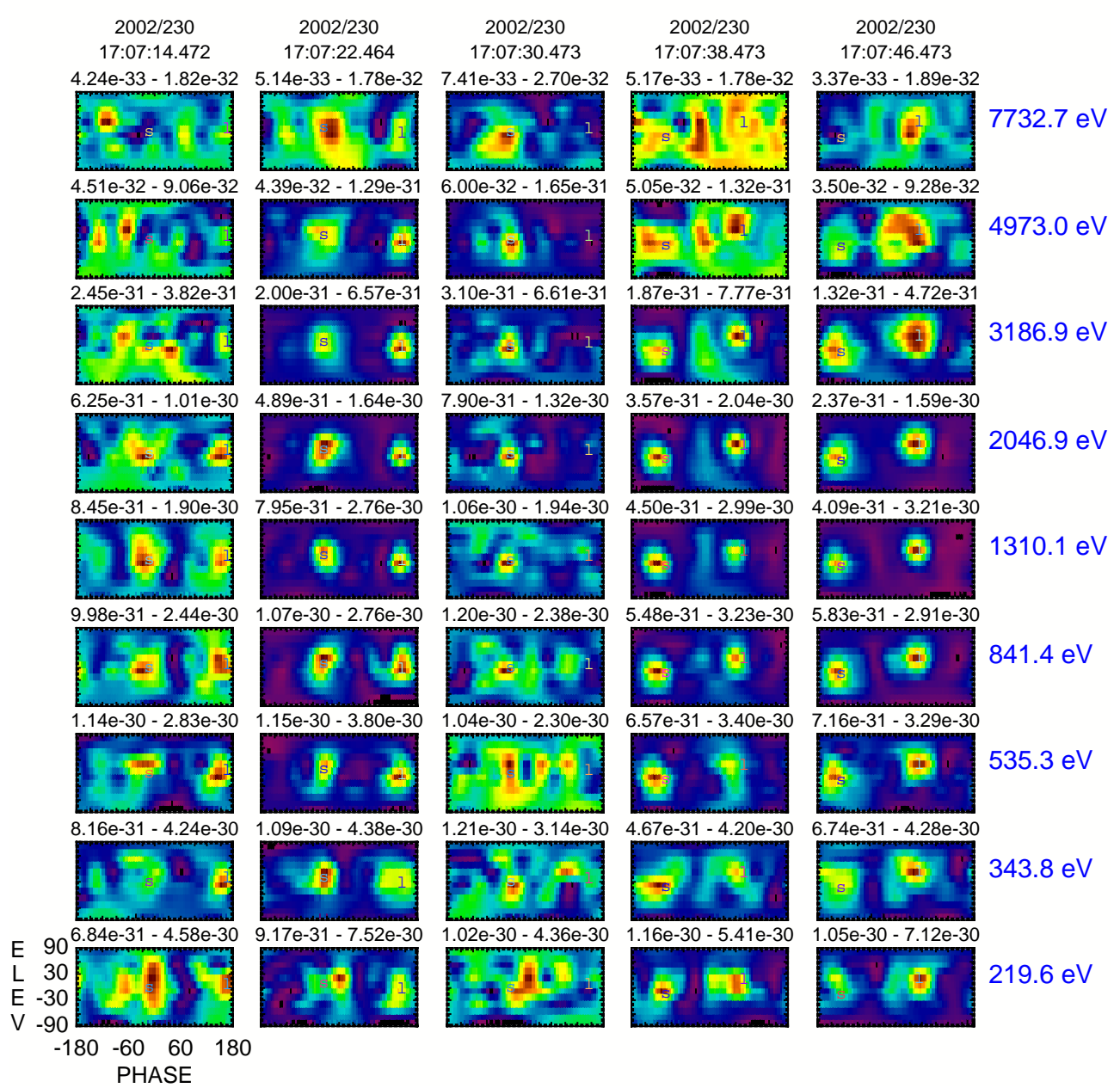

Fig. 4. A set of phi/theta plots of the eVDF in units of $\mathrm{s}^{3} \mathrm{~cm}^{-6}$ during the first vorticity enhancement in event 2 . Each column shows data from multiple energy steps from a single eVDF. The energy step of each row is shown at the extreme right. The beginning accumulation time of each eVDF is shown at the top of each column and the used scaling range above each plot.The existence of bi-directional beams is clearly seen as localized enhancements in the eVDF centered on both of the magnetic field projections (circle and triangle).

\section{Discussion}

Vorticity in the plasma sheet occurs within regions of enhanced bulk flow. With the exception of the three ion diffusion region crossings noted in Figs. 1 and 2, the source of the flow is not determined. The flow and the vorticity observed in conjunction with the crossings of the ion diffusion region are assumed to be created in the reconnection process. Observations of field-aligned flow are not unusual in the plasma sheet and have been associated with boundary layers, magnetic bubbles, and reconnection (Frank et al., 1996; Birn et al., 2004; Nakamura et al., 2006; Åsnes et al., 2008).

Simulations of reconnection in the plasma sheet show that flow around the edges of magnetic islands produce vorticity (e.g., see Matthaeus and Lamkin, 1986). Simulations further show that much of this flow is composed of bi-directional beams that are formed by reflections off of localized potentials or mirroring off of magnetic islands (Onsager et al., 1991; Drake et al., 2006; Egedal et al., 2010). The presence of bi-direction beams in the vicinity of reconnection in the plasma sheet has been observationally reported by a number of authors (e.g., Nakamura et al., 2006; Åsnes et al., 2008).

The fact that the ion diffusion regions seen in Figs. 1 and 2 are embedded within regions of vorticity appears to conform expectations from simulations. The extent of the overshoot in the vorticity on day 299 where it extends more than $30 \mathrm{~min}$ beyond the crossing of the ion diffusion region is somewhat surprising. As with any parameter constructed from spatial derivatives, however, one must be aware of spatial aliasing, which can at times stretch the measured signature. 


\section{CLUSTERII.CLUSTER-1.PEACE.3DX1.CPXP1H $2003229(1051.6 \mathrm{eV})$}

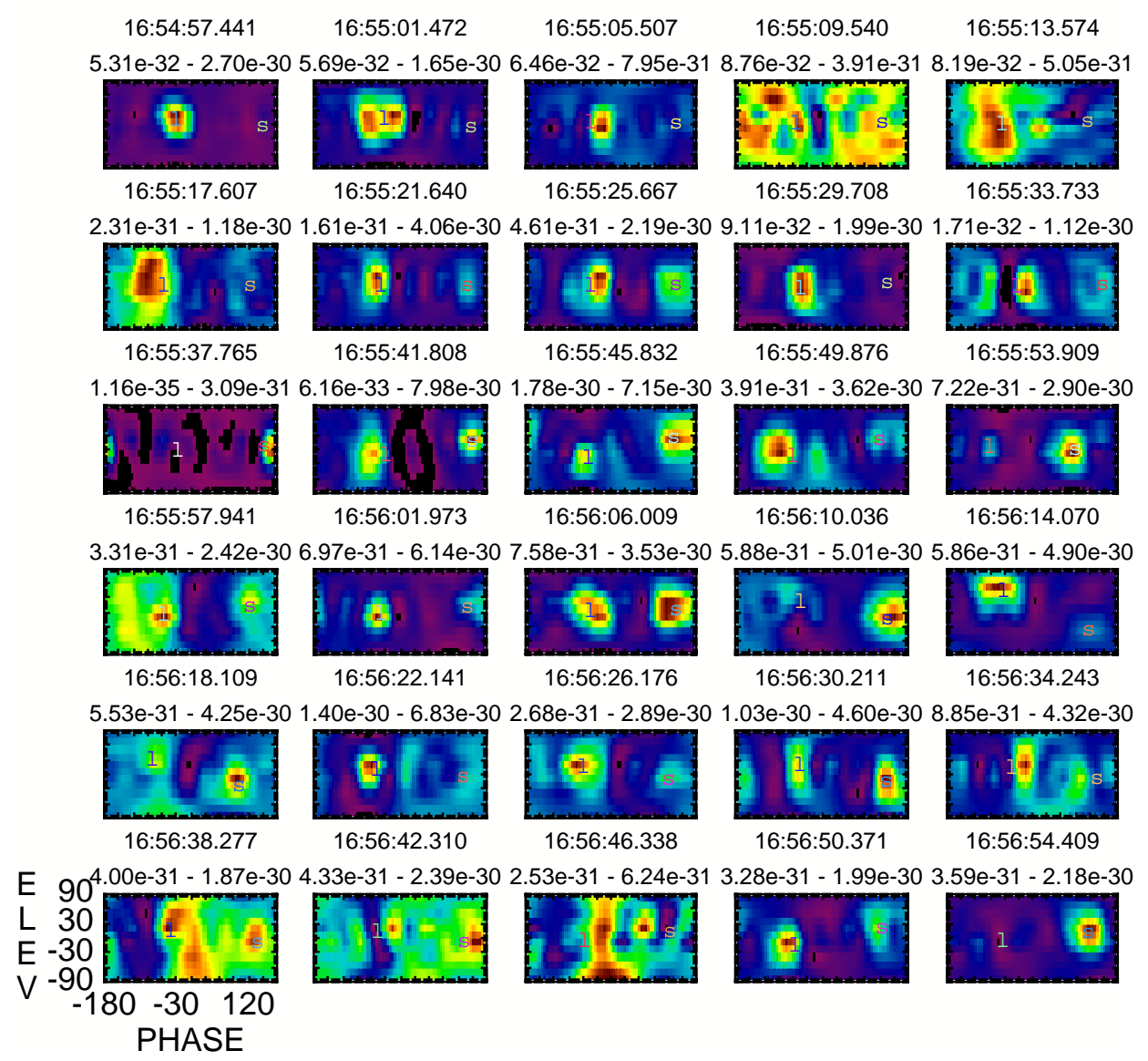

Fig. 5. A set of phi/theta of the eVDF in units of $\mathrm{s}^{3} \mathrm{~cm}^{-6}$ during a period of enhanced vorticity on day 229 of 2003 . The plots show cuts through successive eVDFs at a fixed energy step $(1051 \mathrm{eV})$. The start accumulation time of each eVDF and the applied scaling range is shown above each plot. The figure illustrates the rapid switching between bi-directional and single beams.

Spatial aliasing is caused by the spacecraft separation and generally occurs when the spacecraft cross a quasi-stationary region of enhanced velocity. The amount of stretching is then proportional to the spacecraft separation and velocity. As the spacecraft move across the region, non-zero gradients in the velocity are measured within the spacecraft constellation volume beginning when the first spacecraft enters the region and ending when the last spacecraft exits it. A simple backof-the-envelope calculation using the spacecraft separations and velocities during the ion diffusion region crossings suggests that this could increase the vorticity signature by up to $40 \mathrm{~min}$.

The short overshoots and undershoots in the vorticity surrounding the ion diffusion region crossings on day 230 suggest that the regions of velocity responsible for the observed vorticity are not quasi-stationary, that is they are considerably shorter than can be accounted for by the transition of the spacecraft through the region. This is confirmed in Fig. 6, which shows two sets of plots covering the time of the second crossing of the ion diffusion region in Fig. 1. The top four panels in the left-hand set of plots show the total electron velocity (black) and temperature (red) from each of the four Cluster spacecraft while the bottom panel shows the measured vorticity. The right-hand set of plots shows the spherical components of the magnetic field in GSM at each spacecraft in the top four panels and the magnitude in the lower panel.

The sudden, near simultaneous onset (within 1 or 2 spin periods) in velocity at three of the four spacecraft just prior to entering the ion diffusion region points to this as a temporal event. The cause may result from either burstiness in the reconnection process which would be consistent with the burstiness and irregularity of reconnection associated flows reported by Matthaeus and Lamkin (1986) or from 


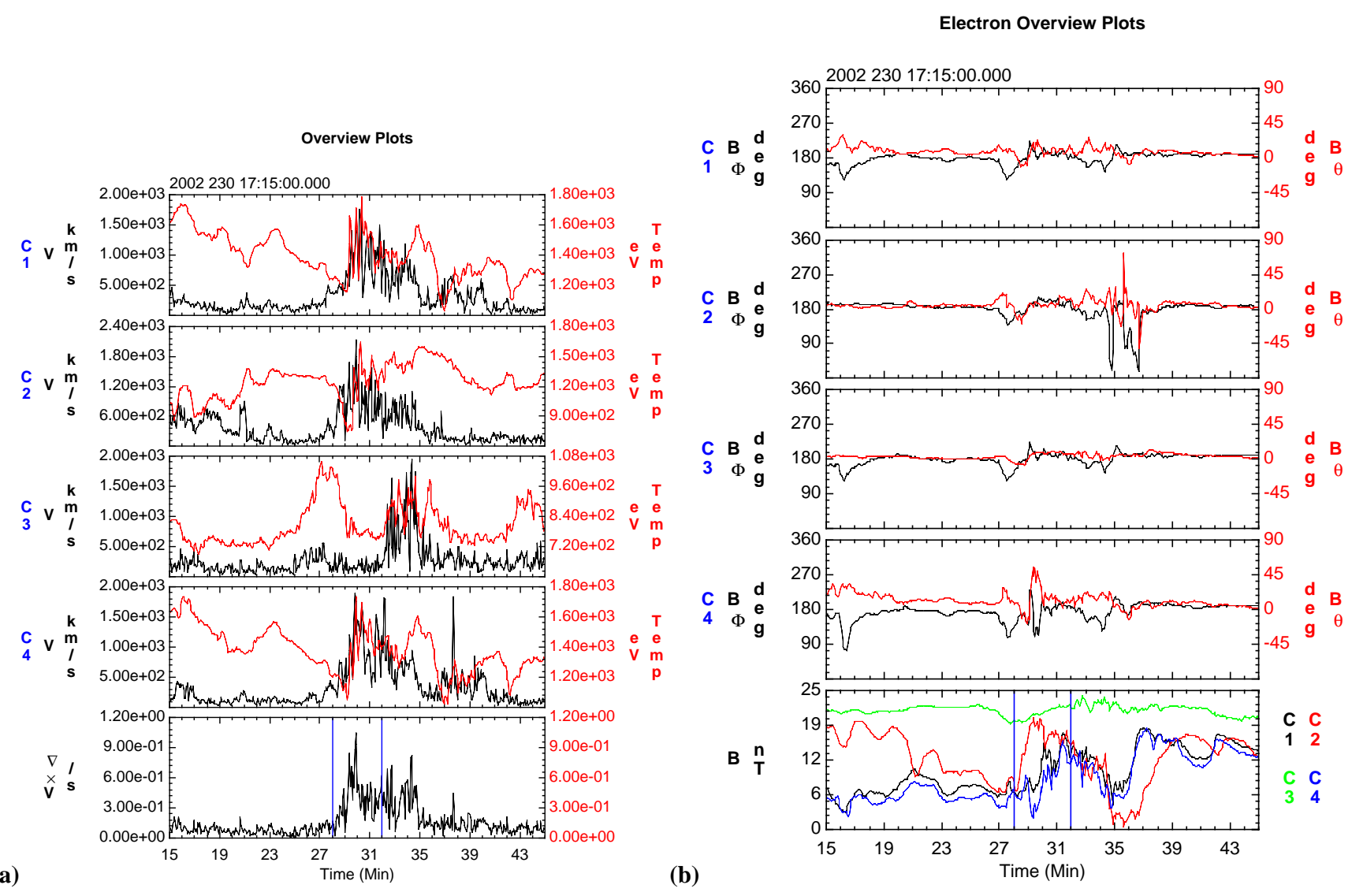

Fig. 6. Comparison of the total electron velocity and temperature and the magnetic field signatures from all four spacecraft across the vorticity burst associated with the second ion diffusion region seen in Fig. 1.

topological changes in the magnetic field that cause the spacecraft to be rapidly enveloped in the velocity changes. The initial onset is not seen by $\mathrm{C} 3$, which is the southernmost spacecraft in the constellation. However, it does see a similar increase in the velocity at about the time that the spacecraft exits the ion diffusion region. The change in velocity does not appear to be caused by any large-scale local change in the magnetic field. In conjunction with this increase, the other three spacecraft show a decrease in the magnitude of the velocity, but still record a large overall bulk flow. The close resemblance of the flow both in magnitude and duration to the flows seen by the other three spacecraft within the ion diffusion region leads one to suspect that this event is also associated with reconnection. It is this flow, together with that seen at the other spacecraft, that leads to the overshoot in the vorticity beyond the end of the ion diffusion region. The velocity falls to background levels at all spacecraft near 17:35 UT, which reinforces the overall observation that the velocity enhancement is basically temporal. The transient nature of the observations means that the velocity must at least extend a distance of several thousand kilometers - large enough to encompass the entire Cluster constellation.
Figure 7 reproduces Fig. 6 but for the interval of vorticity between 09:15 and 09:50 UT seen in Fig. 2. There are only minor differences between the times when the spacecraft first see the velocity region and when they exit the region near 09:50 UT within two spins of one another. This again suggests that the observations are temporal rather than spatial. Between entering and exiting the region, however, the velocity is much more sporadic than what is seen in Fig. 7. It is easy to see the spatial variation in the flow and it is this spatial variation that is responsible for the measured vorticity.

While Eastwood et al. (2010) identify a crossing of the ion diffusion region near the start of the burst in vorticity, the observed overshoot is most likely reconnection related. The time period is much more dynamic magnetically than the time period shown in Fig. 6. There are multiple neutral sheet crossings seen in the spacecraft especially in $\mathrm{C} 4$ between 09:18 and 09:28 UT. These $\mathrm{x}$-line crossings are, if not also crossings of the ion diffusion region, probably place the spacecraft in close proximity to where the electron flows around the magnetic islands are still prominent. 

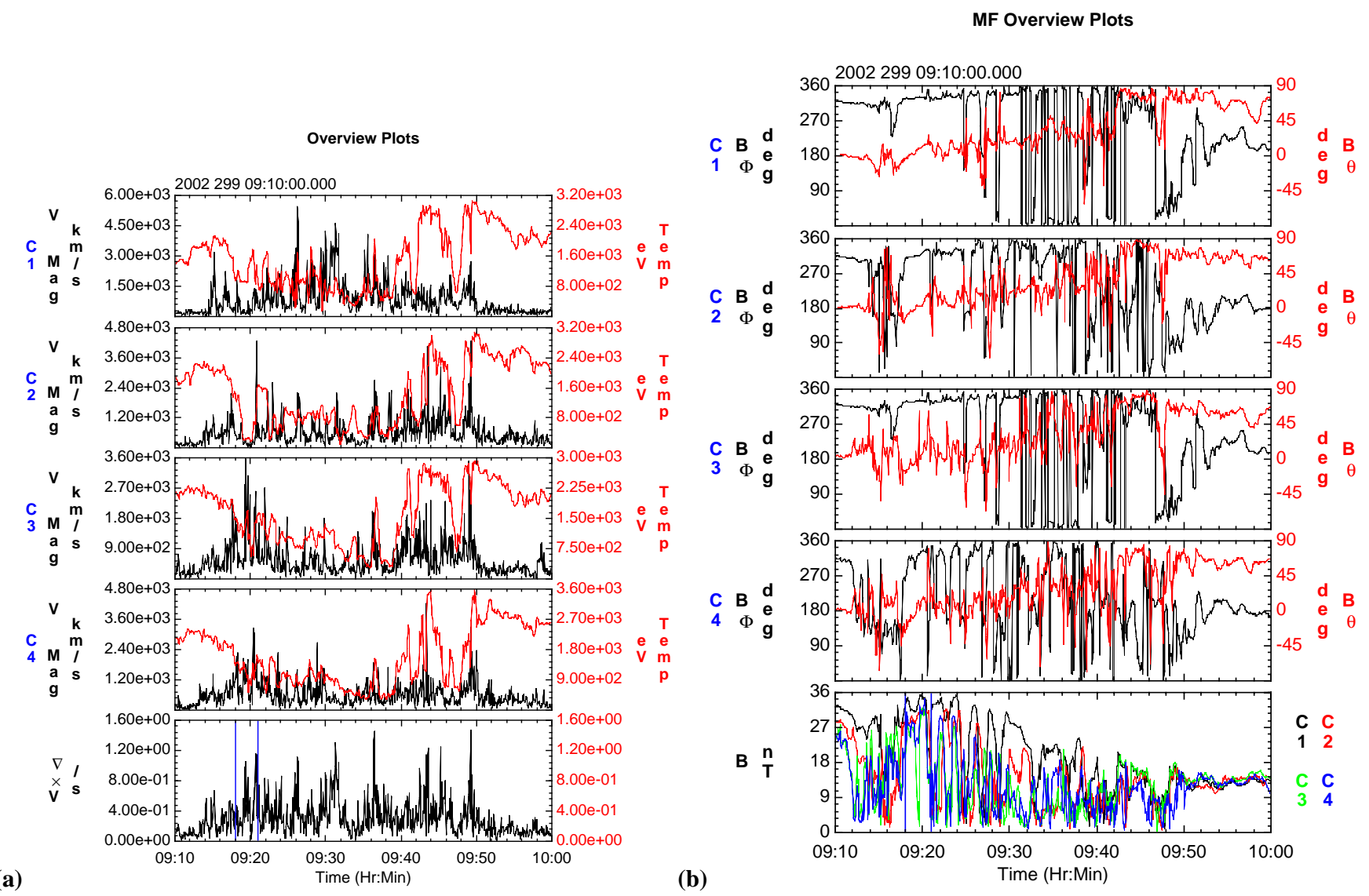

Fig. 7. Comparison of the total electron velocity and temperature and the magnetic field signatures from all four spacecraft across the vorticity burst seen in Fig. 2.

\section{Conclusions}

We have shown that electron vorticity is a common feature in the inner plasma sheet. From the three identified reconnection events, vorticity does appear to be a sensitive indicator of times when the spacecraft are, at least, in the approximate vicinity of reconnecting magnetic fields. This would explain why there are not more correlations of crossings of the ion diffusion regions in the Eastwood et al. (2010) study with the observed bursts in vorticity seen here. Many of the bursts seen may not be associated with a full crossing of the ion diffusion region or simply lack the full set of signatures in the ion data that qualified the event as being identified as an ion diffusion region. While vorticity is certainly associated with $\mathrm{x}$-line reconnection events, we cannot, however, at this time make the definitive claim that all, or even most, of the observed vorticity is caused by reconnection.

Acknowledgements. The authors would like to acknowledge the work and role the Cluster Active Archive (CAA) and thank the EFW, WHISPER and FGM teams for providing the data used in this study. We would also like to acknowledge the PEACE team at MSSL. CG would like to acknowledge support from NASA Grant
NNX10AQ46G and WHM would like to acknowledge support from NASA Grant NNX09AG31G.

Topical Editor I. A. Daglis thanks J. Burch and another anonymous referee for their help in evaluating this paper.

\section{References}

Åsnes, A., Taylor, M. G. G. T., Borg, A. L., Lavraud, B., Friedel, W. H., Escoubet, C. P., Laasko, H., Daly, P., and Fazakerley, A. N.: Multi-spacecraft observations of electron beam in reconnection region, J. Geophys. Res., 113, A07S30, doi:10.1029/2007JA012770, 2008.

Birn, J., Raeder, J., Wang, Y. L., Wolf, R. A., and Hesse, M.: On the propagation of bubbles in the geomagnetic tail, Ann. Geophys., 22, 1773-1786, doi:10.5194/angeo-22-1773-2004, 2004.

Drake, F. M., Swisdak, M., Che, H., and Shay, M. A.: Electron acceleration from contracting magnetic islands during reconnection, Nature Lett., 443, 553-556, doi:10.1038/nature05116, 2006.

Eastwood, J. P., Phan, T. D., Øieroset, M., and Shay, M. A.: Average properties of the magnetic reconnection ion diffusion region in the Earth's magnetotail: The 2001-2005 Cluster obser- 
vations and comparison with simulations, J. Geophys. Res., 115, A08215, doi:10.1029/2009JA014962, 2010.

Egedal, J., Lê, A., Chen, L. J., Lefebvre, B., Daughton, W., and Fazakerley, A.: Cluster observation of bidirectional beams caused by electron trappiung durring antiparallel reconnection, J. Geophys. Res., 115, A03214, doi:10.1029/2009JA014650, 2010.

Frank, L., Paterson, W., Ackerson, K., Kokubun, S., and Yamamoto, T.: Plasma velocity distributions in the near-Earth plasma sheet: A first look with the Geotail spacecraft, J. Geophys. Res., 101, 10627-10637, 1996.

Gurgiolo, C., Goldstein, M. L., Viñas, A. F., and Fazakerley, A. N.: First measurements of electron vorticity in the foreshock and solar wind, Ann. Geophys., 28, 2187-2200, doi:10.5194/angeo28-2187-2010, 2010.

Johnstone, A. D., Alsop, C., Gurge, S., Carter, P. J., Coates, A. J., Coker, A. J., Fazakerley, A. N., Grande, M., Gowen, R. A., Gurgiolo, C., Hancock, B. K., Narheim, B., Preece, A., Sheather, P. H., Winningham, J. D., and Woodcliffe, R. D.: PEACE: A plasma electron and current experiment, Space Sci. Rev., 79, 351-398, 1997.
Matthaeus, W. H.: Reconnection in two dimensions: Localization of vorticity and current near the X-points, Geophys. Res. Lett., 9, 660-663, 1982.

Matthaeus, W. H. and Lamkin, S. L.: Turbulent Magnetic Reconection, Phys. Fluids, 8, 2513-2534, 1986.

Nakamura, R., Baumjohann, W., Asano, Y., Runov, A., Balogh, A., Owen, C. J., Fazakerley, A. N., Fujimoto, M., Klecker, B., and Réme, H.: Dynamics of thin current sheets associated with magnetotail reconnection, J. Geophys. Res., 111, A11206, doi:10.1029/2006JA011706, 2006.

Onsager, T. G., Thomsen, M. F., Elphic, R. C., and Gosling, J. T.: Model of electron and ion distributions in the plasma sheet boundary layer, J. Geophys. Res., 96, 20999-21011, doi:10.1029/91JA01983, 1991.

Robert, P., Roux, A., Harvey, C. C., Dunlop, M. W., Daly, P. W., and Glassmeier, K.-H.: Tetrahedron Geometry Factors, in: Analysis methods for multi-spacecraft data, edited by: Paschmann, G. and Daly, P. W., p. 323, ESA Publications Division, Keplerlaan 1, 2200 AG Noordwijk, The Netherlands, 1998. 